\title{
ON THE TRIVIALITY OF CERTAIN WHITEHEAD GROUPS
}

Article in Mathematical Proceedings of the Royal Irish Academy · December 2007

DOI: 10.3318/PRIA.2007.107.2.183

CITATION

1

1 author:

Some of the authors of this publication are also working on these related projects:
READS

5
Nicolas Grenier-Boley

Université de Rouen

14 PUBLICATIONS 18 CITATIONS

SEE PROFILE 


\title{
On the triviality of certain Whitehead groups
}

\author{
N. Grenier-Boley
}

March 19, 2006

\begin{abstract}
Let $K$ be a field of arbitrary characteristic and $q$ be a prime number different from the characteristic of $K$. If $A$ is a central simple algebra over $K$ whose index is a power of $q$, we show the triviality of the Whitehead groups $\operatorname{SK}_{1}(A)$ and $\operatorname{USK}_{1}(A)$ when the cohomological $q$-dimension of $K$ is at most 2 . We give a global version of this result and indicate what can be done in the case where the index of the algebra is a power of the characteristic of the field. Triviality results of the Whitehead group $\mathrm{K}_{1} \operatorname{Spin}(A)$ are easily derived.
\end{abstract}

Mathematics subject classification (2000): 19Bxx.

Keywords: Central simple algebra, Whitehead group.

\section{Introduction}

Let $K$ be a field of arbitrary characteristic. If $A$ is a (finite dimensional) central simple algebra over $K$ and if $\operatorname{Nrd}_{A / K}$ is the reduced norm map from $A$ to $K$, we set

$$
\mathrm{SL}_{1}(A)=\left\{a \in A^{*} \mid \operatorname{Nrd}_{A / K}(a)=1\right\},
$$

and we denote by $\left[A^{*}, A^{*}\right]$ the commutator subgroup of $A^{*}$. By definition, the reduced Whitehead group of $A$ is the factor group

$$
\operatorname{SK}_{1}(A)=\mathrm{SL}_{1}(A) /\left[A^{*}, A^{*}\right]
$$

It was conjectured (independently) by Tannaka and $\operatorname{Artin}$ that $\operatorname{SK}_{1}(A)=1$ when $A$ is different from $M_{2}\left(\mathbb{F}_{2}\right)$ and $M_{2}\left(\mathbb{F}_{3}\right)$. This conjecture is part of a more general conjecture due to Kneser and Tits concerning simply connected algebraic groups: more precisely, Tannaka-Artin's Conjecture is equivalent to Kneser-Tits's Conjecture for algebraic groups of type $A_{n}$, see [17]. It turns out that these conjectures are false in general by results of Platonov in [11]. In a series of papers (see references (8) to (14) in Yanchevskiı̌'s paper [21]), Platonov consequently builds a reduced $K$-theory and highlights important connections between reduced Whitehead groups and algebraic groups. However, the reduced Whitehead group is trivial in many cases and it is still interesting to find sufficient conditions over $K$ or over $A$ to guarantee this triviality. For example, an important result due to Yanchevskil asserts that $\operatorname{SK}_{1}(A)$ is trivial whenever the base field is a $C_{2}^{0}$-field (see Theorem 2.6). 
Since 1973, Platonov and Yanchevskiu have been developing a reduced unitary $K$-theory in which the central object is the reduced unitary Whitehead group $\operatorname{USK}_{1}(A)$. This group is an analog of the reduced Whitehead group for central simple algebras endowed with an unitary involution and its triviality is equivalent to Kneser-Tits's Conjecture for unitary groups. In [12], they show that this group is not always trivial. Nevertheless, Yanchevskil proves that this group is trivial if the center of $A$ is a $C_{2}^{0}$-field (see Theorem 2.11).

In the case where the central simple algebra is endowed with a symplectic involution, the ana$\log$ of the reduced Whitehead group is the group $\mathrm{K}_{1} \operatorname{Spin}(A)$. In [9], Monastyrnyı̆ and Yanchevskiu show that this group is not trivial in general even if it is still the case over $C_{2}^{0}$-fields.

Many results concerning these three types of Whitehead groups are available in the literature and some of them are recalled in Section 2.

In $[15, \S 24]$, as a consequence of their works about norm residue homomorphisms, Merkurjev and Suslin prove that the cohomological $p$-dimension of a field $K$ is at most 2 if and only if $K$ is a $C_{2}^{0}$-field locally at $p$, if $p$ is different from the characteristic of the field (see Theorem 3.1). In particular, if $F$ is a perfect field, the cohomological dimension of $F$ is at most 2 if and only if $F$ is a $C_{2}^{0}$-field (see Corollary 3.2). Section 3 is dedicated to the recall of these results and focuses on a proof of this last fact.

All these results allow us to think that there are local cohomological criteria for the above Whitehead groups to be trivial. Our main result asserts that this is true modulo a restriction on the index of the algebras:

Theorem 1.1. Let $K$ be a field of characteristic $p$ (which can be zero) and $q$ be a prime number different from $p$. Suppose that $\operatorname{cd}_{q}(K) \leq 2$ and that $A$ is a central simple algebra over $K$ whose index is q-primary. Then the Whitehead groups $\operatorname{SK}_{1}(A)$ and $\operatorname{USK}_{1}(A)$ are trivial.

In Section 4, we prove preliminary results. First we are interested in scalar extension results (see Lemma 4.3). Then we adapt a result given by Bayer-Fluckiger and Serre in [2] to construct a perfect field satisfying certain properties starting from any field and from any prime number different from the characteristic of the field (see Proposition 4.4). In Section 5, we give the proof of Theorem 1.1. As a consequence, we obtain a triviality result for the group $\mathrm{K}_{1} \operatorname{Spin}(A)$ stated as Corollary 5.2.

In [6], Gille defines the separable $p$-dimension of a field based on the notion of $p$-dimension initially defined by Kato. Using this notion, he completes Merkurjev and Suslin's result above in the case where the degree of the algebra is a power of the characteristic of the field (see Theorem 5.4). In the last part of Section 5, we indicate that, consequently to Gille's result, Theorem 1.1 is still true when $q=p=\operatorname{char}(F)$ if we suppose that the separable $p$-dimension of $F$ is at most 2 (see Corollary 5.5). Finally, we obtain the following global result:

Corollary 1.2. (1) Suppose that $K$ is a field whose separable dimension is at most 2. Then for any central simple algebra $A$ over $K$, the Whitehead groups $\operatorname{SK}_{1}(A)$ and $\operatorname{USK}_{1}(A)$ are trivial.

(2) Suppose that $K$ is a field whose separable 2-dimension is at most 2. Then for any central simple algebra $A$ over $K$, the Whitehead group $\mathrm{K}_{1} \operatorname{Spin}(A)$ is trivial. 


\section{Whitehead groups}

This Section presents some basic properties and triviality results for the Whitehead groups under study. Other standard facts and results about the reduced Whitehead group can be found in $[5, \S 23]$ or in $[13$, Chapter $4, \S 2]$. Concerning reduced unitary Whitehead groups, we refer to [21], [22], [23], [12] or [13, Chapter 4, §3] for more precise statements. Standard references for the group $\mathrm{K}_{1} \operatorname{Spin}(A)$ are [9] and [23].

\subsection{The group $\mathrm{SK}_{1}(A)$}

\section{Basic properties}

Let $K$ be a field and $A$ be a central simple algebra over $K$. From now on, $G_{K}$ will denote the absolute Galois group of $K$. If $p$ is a prime number, $\operatorname{cd}_{p}(K)$ is the cohomological $p$-dimension of $G_{K}$ and $\operatorname{cd}(K)$ is the cohomological dimension of $G_{K}$. We refer to [14, Chapitre I, §3] for precise results about Galois cohomology.

Recall that the reduced Whitehead group $\operatorname{SK}_{1}(A)$ is the abelian group $\operatorname{SL}_{1}(A) /\left[A^{*}, A^{*}\right]$. For split algebras, this group is known:

Proposition 2.1 (Wang). Let $n \geq 1$ and suppose that $n \neq 2$ in the case where $K=\mathbb{F}_{2}$ or $K=\mathbb{F}_{3}$. Then we have $\operatorname{SK}_{1}\left(M_{n}(K)\right)=1$.

Proof. See $[5, \S 20$, Theorem 4] for a more general result.

Remark 2.2. We easily show that $\operatorname{SK}_{1}\left(M_{2}\left(\mathbb{F}_{2}\right)\right)$ is a cyclic group of order 2 and that $\operatorname{SK}_{1}\left(M_{2}\left(\mathbb{F}_{3}\right)\right)$ is a cyclic group of order 3 .

Convention 2.3. From now on, when speaking about the reduced Whitehead group of $A$, we will always implicitly assume that $A \neq M_{2}\left(\mathbb{F}_{2}\right), M_{2}\left(\mathbb{F}_{3}\right)$.

By means of the theory of Dieudonné's determinants, one proves that the reduced Whitehead group of $A$ only depends on the Brauer class of $A$ in $\operatorname{Br}(K)$ (see $[5, \S 20]$ or [13, Chapter $2, \S 4]$ ). Indeed

$$
\mathrm{SK}_{1}(A) \simeq \mathrm{SK}_{1}(D)
$$

where $D$ is a division algebra Brauer-equivalent to $A$. Consequently, the exponent of $\operatorname{SK}_{1}(A)$ is the same as the exponent of $\mathrm{SK}_{1}(D)$. It can be shown that the exponent of $\operatorname{SK}_{1}(A)$ divides the index of $A$ (apply Lemma 4.1 to a maximal commutative subfield of $D$ ). Moreover, every division algebra $D$ of degree $\prod_{i=1}^{n} p_{i}^{n_{i}}$ (where the $p_{i}$ 's are distinct prime numbers) can be decomposed as $D=D_{1} \otimes_{K} \cdots \otimes_{K} D_{n}$ where each $D_{i}$ is a division algebra of degree $p_{i}^{n_{i}}$ (see [5, $\S 9$, Corollary 11]). With the same notations,

$$
\mathrm{SK}_{1}(D) \simeq \coprod_{i=1}^{n} \mathrm{SK}_{1}\left(D_{i}\right)
$$

(see $[5, \S 23$, Lemma 6] or $[13$, Chapter $4, \S 2.2$, Theorem 1]) hence it suffices to study reduced Whitehead groups of division algebras whose degree is $p$-primary where $p$ is a prime number. 


\section{Triviality results}

Whereas the reduced Whitehead group is not always trivial, this result is true over a great number of fields. Examples of such fields are $p$-adic fields by a result of Nakayama and Matsushima (see [10]) and algebraic number fields by a result due to Wang (see [18, Theorem p. 329]). These two results contributed to the fact that people expected Tannaka-Artin's conjecture to be true. In this context $C_{2}^{0}$-fields are of great importance:

Definition 2.4. A field $K$ is a $C_{2}^{0}$-field if for any finite field extension $E / K$ and for any finitedimensional division algebra $D$ with center $E$, the reduced norm map $\operatorname{Nrd}_{D / E}: D^{*} \rightarrow E^{*}$ is surjective.

Remark 2.5. One can see that $C_{2}$-fields are $C_{2}^{0}$-fields (see [13, Chapter II, $\left.\S 4.3\right]$ ). The class of $C_{2}^{0}$-fields is strictly bigger that the class of $C_{2}$-fields as it contains the field $\mathbb{Q}_{2}$ which is not a $C_{2}$-field by a result of Terjanian (see [14, p. 98]).

An important triviality result is the following:

Theorem 2.6 (Yanchevskiı̌). If $K$ is a $C_{2}^{0}$-field, then $\operatorname{SK}_{1}(A)=1$.

Proof. See [20, Theorem p. 492].

As a consequence, the reduced Whitehead group of any central simple algebra over an algebraic function field is trivial (such a field is a $C_{2}$-field, see [14, Chapitre II, §4.5]). From the previous results, it follows that this property also holds over global fields.

By a theorem of Wang, $\operatorname{SK}_{1}(A)$ is trivial if the index of $A$ is squarefree (see [18]). This result is no longer true if the index of the algebra is not squarefree: from a field $K$ of characteristic different from 2 containing a primitive fourth root of unity, one can construct another field (of cohomological dimension strictly greater than 3 ) over which there exists a biquaternion algebra $A$ with $\operatorname{SK}_{1}(A) \neq 1$ (see [7, Example 17.23]). Note thatThis construction Platonov's original counterexample to Tannaka-Artin's conjecture was built from a biquaternion algebra over a field $K$ satisfying $\operatorname{cd}(K) \geq 4$. However,

Theorem 2.7 (Rost). If $\operatorname{cd}(K) \leq 3$, then $\operatorname{SK}_{1}(A)=1$ for any biquaternion algebra $A$ over $K$.

Proof. See [7, Chapter 17] or Merkurjev's proof in [8, Corollary p.76].

More generally, in [16], Suslin conjectures the following:

Conjecture 2.8 (Suslin). If $\operatorname{cd}(K) \leq 3, \operatorname{SK}_{1}(A)=1$. 


\subsection{The group $\operatorname{USK}_{1}(A)$}

In this Subsection, we suppose that $A$ is endowed with an unitary involution $\sigma$. In this case, we will always denote by $F$ the subfield of central invariant elements under $\sigma$.

\section{Basic properties}

The reduced unitary Whitehead group of $A$ is, by definition

$$
\left(\operatorname{USK}_{1}\right)_{\sigma}(A)=\Sigma_{\sigma}^{\prime}(A) / \Sigma_{\sigma}(A),
$$

where $\Sigma_{\sigma}(A)\left(\right.$ resp. $\left.\Sigma_{\sigma}^{\prime}(A)\right)$ is the subgroup of $A^{*}$ generated by the elements that are symmetric (resp. that have symmetric reduced norm) with respect to $\sigma$. One can define an equivalence relation $\sim$ on the set of unitary involutions over $A$ by putting $\left.\sigma \sim \theta \Longleftrightarrow \sigma\right|_{K}=\left.\theta\right|_{K}$. We easily see that the definitions of $\left(\operatorname{USK}_{1}\right)_{\sigma}(A), \Sigma_{\sigma}(A)$ and $\Sigma_{\sigma}^{\prime}(A)$ only depend on the class of $\sigma$ modulo the relation $\sim$, see $[19$, Lemma 1]. From now on, these groups will then be respectively denoted by $\operatorname{USK}_{1}(A), \Sigma(A)$ and $\Sigma^{\prime}(A)$.

It turns out that the reduced unitary Whitehead group of $A$ has many properties in common with the reduced Whitehead group. For example, properties (1) and (2) are still true when replacing $\mathrm{SK}_{1}$ by $\mathrm{USK}_{1}$ (see [19, Lemma2, Lemma 3] and [21, Proposition 2.7]) and its exponent still divides the index of $A$. Again, it suffices to study the reduced unitary Whitehead groups of divison algebras whose degree is $p$-primary where $p$ is a prime.

We have:

Proposition 2.9 (Yanchevskiǔ). $\left[A^{*}, A^{*}\right] \subseteq \Sigma(A)$.

Proof. See [19, Lemma 2].

This result implies that the group $\operatorname{USK}_{1}(A)$ is abelian but can also be used to deduce a link between $\operatorname{SK}_{1}(A)$ and $\operatorname{USK}_{1}(A)$ as we now show. If $x \in \Sigma^{\prime}(A)$, then we can write $x=\sigma(x) a$ where $a \in \mathrm{SL}_{1}(A)$. Thus, by Proposition 2.9, the canonical surjection from $\operatorname{SL}_{1}(A)$ to $\operatorname{SK}_{1}(A)$ induce a group homomorphism $\Phi: \operatorname{USK}_{1}(A) \rightarrow \mathrm{SK}_{1}(A): x \mapsto \sigma(x)^{-1} x$. This homomorphism is of great importance when proving triviality results:

Lemma 2.10 (Yanchevskiı)). The exponent of ker $\Phi$ divides 2. In particular, if the index of $A$ is odd, $\Phi$ is injective.

Proof. The proof can be found in [21, Lemma p. 183]. Suppose that $x \in \Sigma^{\prime}(A)$ and write $x=\sigma(x) a$ where $a \in \mathrm{SL}_{1}(A)$. If $x \in \operatorname{ker} \Phi$ then $a \in\left[A^{*}, A^{*}\right]$. By Proposition 2.9,

$$
x^{2}=x \sigma(x) a \in \Sigma(A) .
$$

As the exponent of $\operatorname{USK}_{1}(A)$ is odd, we obtain that $x \in \Sigma(A)$.

\section{Triviality results}

The reduced unitary Whitehead group of a central simple algebra is not trivial in general (this was first proved by Platonov and Yanchevskii, see [12]). However, one can show that this group is trivial over any global field (see [12]), when the index of $A$ is a prime number (see [19, Lemma 5] or [4, Théorème p. 66] for a more general result) and that Theorem 2.6 has an analog: 
Theorem 2.11 (Yanchevskiı̌). If $K$ is a $C_{2}^{0}$-field, then $\operatorname{USK}_{1}(A)=1$.

Proof. See [19, Theorem 1].

\subsection{The group $K_{1} \operatorname{Spin}(A)$}

In this Subsection, the central simple algebra $A$ is supposed to be endowed with a symplectic involution $\sigma$. Let

$$
R(A)=\left\{a \in A^{*} \mid \operatorname{Nrd}_{A / K}(a) \in F^{* 2}\right\} .
$$

The involution $\sigma$ being symplectic, $\Sigma_{\sigma}(A) \subset R(A)$ (see [7, Proposition 2.9]) thus we may define

$$
\mathrm{K}_{1} \operatorname{Spin}(A)=R(A) / \Sigma_{\sigma}(A)\left[A^{*}, A^{*}\right] .
$$

This group does not depend on the choice of $\sigma$, is 2-torsion abelian and is not trivial in general (see [9]). However, this group is trivial if $\operatorname{deg} A \leq 4$ by a result of Yanchevskiu (see [7, Proposition 17.28]) or if $K$ is a $C_{2}^{0}$-field (see Corollary 5.2 for a similar proof).

\section{About a result of Merkurjev and Suslin}

The purpose of this Section is to give a proof of Corollary 3.2 which is a consequence of the following deep result of Merkurjev and Suslin.

Theorem 3.1 (Merkurjev-Suslin). Let $K$ be a field and $p$ be a prime number different from the characteristic of $K$. Then the following are equivalent:

(1) $\operatorname{cd}_{p}(K) \leq 2$.

(2) For any finite field extension $E / K$ and for any central simple algebra $A$ over $E$, if the degree of $A$ is a power of $p$ then the reduced norm map $\operatorname{Nrd}_{A / K}: A^{*} \rightarrow E^{*}$ is surjective.

Proof. See [15, Theorem 24.8].

The following Corollary was also stated by Merkurjev and Suslin and will be a main tool in the proofs of our results:

Corollary 3.2 (Merkurjev-Suslin). If $K$ is a perfect field, the following are equivalent:

(1) $\operatorname{cd}(K) \leq 2$.

(2) $K$ is a $C_{2}^{0}$-field.

Proof. This Corollary is stated in [15, Corollary 24.9] as a direct consequence of Theorem 3.1. We give a proof of this fact.

Suppose first that $K$ is a $C_{2}^{0}$-field. Let $p$ be a prime number different from $\operatorname{char}(K), E / K$ be a finite field extension and $A$ be a central simple algebra over $E$ whose degree is a power of $p$. By Wedderburn's Theorem, one can identify $A$ and $M_{n}(D)$ where $D$ is a division algebra over $E$. As $K$ is a $C_{2}^{0}$-field, the map $\operatorname{Nrd}_{D / E}: D^{*} \rightarrow E^{*}$ is surjective hence also $\operatorname{Nrd}_{A / E}: A^{*} \rightarrow E^{*}$ using Dieudonné's determinant (see [5, §22, Theorem 3]). By Theorem 3.1, $\operatorname{cd}(K) \leq 2$. 
Suppose now that $\operatorname{cd}(K) \leq 2$ and consider a finite field extension $E / K$ and a division algebra $D$ over $E$. One can decompose $D$ as follows

$$
D \simeq D_{1} \otimes_{E} \cdots \otimes_{E} D_{s}
$$

where the $D_{i}$ 's are division algebras over $E$ with $\operatorname{deg}\left(D_{i}\right)=p_{i}{ }^{f_{i}}$ such that the $p_{i}$ 's are distinct prime numbers and $\operatorname{deg}(D)=\prod_{i=1}^{s} p_{i}{ }^{f_{i}}$. We claim that $\operatorname{Nrd}_{D_{i} / E}: D_{i}{ }^{*} \rightarrow E^{*}$ is surjective for all $i$ 's. If $p_{i} \neq \operatorname{char}(K)$, this follows from Theorem 3.1 and if $p_{i}=\operatorname{char}(K)$, this is guaranteed by the perfectness of $E$. If $e \in E^{*}$ then, for any $1 \leq j \leq s$, there exists $d_{j} \in D_{j}{ }^{*}$ with $\operatorname{Nrd}_{D_{j} / E}\left(d_{j}\right)=e$ hence, by [5, §22, Theorem 3]), $e^{\prod_{i \neq j} p_{i}^{f_{i}}} \in \operatorname{Nrd}_{D / E}\left(D^{*}\right)$. As $\operatorname{pgcd}\left(\prod_{i \neq j} p_{i}^{f_{i}}, 1 \leq j \leq s\right)=1$ there exist $\lambda_{1}, \cdots, \lambda_{s} \in \mathbb{Z}$ such that

$$
\lambda_{1} \prod_{i \neq 1} p_{i}^{f_{i}}+\cdots+\lambda_{s} \prod_{i \neq s} p_{i}^{f_{i}}=1
$$

and

$$
\operatorname{Nrd}_{D / E}\left(d_{1}^{\lambda_{1}} \otimes \cdots \otimes d_{s}^{\lambda_{s}}\right)=e
$$

thus finishing the proof.

Remarks 3.3. (1) In Corollary $3.2,(2) \Rightarrow(1)$ is true in general. We thus have:

$$
K \text { is a } C_{2} \text {-field } \Rightarrow K \text { is a } C_{2}^{0} \text {-field } \Rightarrow \operatorname{cd}(K) \leq 2 \Rightarrow \operatorname{cd}_{2}(K) \leq 2 \Rightarrow I^{3}(K)=0
$$

where $I(K)$ is the fundamental ideal of the Witt ring of $K$ and $I^{3}(K):=(I(K))^{3}$.

(2) Theorem 3.1 and Corollary 3.2 have many important consequences. For example, Theorem 3.1 shows that Serre's Conjecture II is true for special linear groups and Corollary 3.2 has been used by Bayer-Fluckiger and Parimala to show this Conjecture for special unitary groups, see $[1$, Theorem 5.1.2]).

\section{Preliminary results}

\subsection{Scalar extension}

In this Subsection, $D$ denotes a division algebra over $K$. We state preliminary results with respect to scalar extension to a field.

Lemma 4.1 (Wang). Let $L / K$ be a field extension of degree $m$. If $\alpha \in D$ is such that $\alpha \otimes 1 \in$ $\left[\left(D \otimes_{K} L\right)^{*},\left(D \otimes_{K} L\right)^{*}\right]$ then $\alpha^{m} \in\left[D^{*}, D^{*}\right]$.

Proof. See [18, Lemma 3].

Suppose now that $D$ is endowed with an unitary involution $\sigma$ and that $F$ is, as usual, the subfield of $K$ fixed by $\sigma$. The following Lemma is an analog of Lemma 4.1 for $\mathrm{USK}_{1}$.

Lemma 4.2 (Yanchevskiı). Let $L / K$ be a field extension of degree $m$ containing an element a such that $L=K(a)$ and $[L: F(a)]=2$. If $\beta \in \Sigma^{\prime}(D)$ is such that $\beta \otimes 1 \in \Sigma\left(D \otimes_{K} L\right)$ then $\beta^{m} \in \Sigma(D)$. 
Proof. See [19, Lemma 4].

As a consequence, we obtain:

Lemma 4.3. Let $H / F$ be a field extension of degree $m$ which is linearly disjoint from the extension $K / F$ and let $L$ be the composite of $K$ and $H$. If $\beta \in \Sigma^{\prime}(D)$ is such that $\beta \otimes 1 \in \Sigma\left(D \otimes_{K} L\right)$ then $\beta^{m} \in \Sigma(D)$.

Proof. There exist $a_{1}, \cdots, a_{n}$ such that $H=F\left(a_{1}, \cdots, a_{n}\right)$ and $L=K\left(a_{1}, \cdots, a_{n}\right)$. For $i=$ $1, \cdots, n$, write $H_{i}=F\left(a_{1}, \cdots, a_{i}\right)$ and $L_{i}=K\left(a_{1}, \cdots, a_{i}\right)$. As $K$ and $H$ are linearly disjoint over $F,\left[L_{i}: K_{i}\right]=[K: F]=2$ (see [3, Chapitre V, $\S 2, \mathrm{n}^{\circ} 3$, Proposition 6]). Consequently, the field extensions $L_{i} / K_{i}$ are Galois. The central simple $L$-algebra $D \otimes_{K} L$ is endowed with the unitary involution $\sigma \otimes \theta$, where $\theta$ is the nontrivial automorphism of $L$ over $H$. If $D^{\prime}=D \otimes_{K} L_{n-1}$, there is an $L$-algebra isomorphism $D \otimes_{K} L \simeq D^{\prime} \otimes_{L_{n-1}} L$. Note that, by definition of $\theta,\left.\theta\right|_{L_{n-1}}$ is the nontrivial automorphism of $L_{n-1}$ over $K_{n-1}$. If $\sigma^{\prime}=\left.\sigma \otimes \theta\right|_{L_{n-1}}, \sigma^{\prime} \otimes \theta$ is an unitary involution over $D^{\prime} \otimes_{L_{n-1}} L$. Now $\beta \in \Sigma^{\prime}\left(D^{\prime}\right)$ and $\beta \otimes 1 \in \Sigma\left(D^{\prime} \otimes_{L_{n-1}} L\right)$. By Lemma 4.2, it follows that

$$
(\beta \otimes 1)^{\left[L: L_{n-1}\right]} \in \Sigma\left(D^{\prime}\right)=\Sigma\left(D \otimes_{K} L_{n-1}\right) .
$$

By induction over $n$, we get that $\beta^{m} \in \Sigma(D)$ where $m=\left[L: L_{n-1}\right] \cdots\left[L_{1}: K\right]=[L: K]$.

\subsection{Construction of fields satisfying certain conditions}

In this Subsection, we are interested in constructing fields satisfying certain properties starting from any field. Fix $\bar{K}$ an algebraic closure of $K$ and $K_{\text {sep }}$ a separable closure of $K$. If char $(K)=$ $p \neq 0$, recall that the radicial closure of $K$ in $\bar{K}$ is the subfield of $\bar{K}$ of radicial elements over $K$ denoted by $K^{p^{-\infty}}$. More precisely, $K^{p^{-\infty}}=\bigcup_{e \in \mathbb{N}} K^{p^{-e}}$ where $x \in K^{p^{-e}}$ if and only if $x^{p^{e}} \in K$ : see $[3$, Chapitre $\mathrm{V}, \S 8]$ for more details.

Proposition 4.4. Let $K$ be a field and $q$ be a prime number different from $\operatorname{char}(K)=p$ ( $p$ can possibly be zero). There exists an algebraic extension $K^{\prime} / K$ having the following properties:

(1) $K^{\prime}$ is a filtered union of field extensions of $K$ whose degree is prime to $q$.

(2) $K^{\prime}$ is a perfect field.

(3) $G_{K^{\prime}}$ is a pro-q-group.

Moreover $\operatorname{cd}\left(K^{\prime}\right)=\operatorname{cd}_{q}(K)$.

Proof. When $q=2$, this result is due to Bayer-Fluckiger and Serre in [2, Proposition 2.3.1]. The general case comes from the same kind of arguments which we are going to detail.

Denote by $S_{q}\left(G_{K}\right)$ a $q$-Sylow of $G_{K}$ (which exists in accordance with [14, Chapitre I, $\S 1$, Proposition 3]) and by $K_{q}$ the subfield of $K_{\text {sep }}$ fixed by $S_{q}\left(G_{K}\right)$. Then $K_{q}=\bigcup_{x \in K_{q}} K(x)$. The field extension $K_{q} / K$ is thus a union of field extensions of $K$ whose degree is prime to $q$. The Theorem of the Primitive Element implies that this is a filtered union. If $\operatorname{char}(K)=0$, we choose $K^{\prime}=K_{q}$.

Suppose now that $\operatorname{char}(K)=p \neq 0$ and denote by $K^{\prime}=K_{q}^{p^{-\infty}}$. Then

$$
K^{\prime}=\bigcup_{L \in J} L
$$


where $J$ is the set of finite degree field extensions $L / K$ such that there exist $e \in \mathbb{N}$ and $x \in K_{q}$ with $L \subset(K(x))^{p^{-e}}$. This union is a filtered union. Let $L / K \in J$. Then, there exist $x \in K_{q}$ and $e \in \mathbb{N}$ such that $L \subset(K(x))^{p^{-e}}$. As $x \in K_{q},[K(x): K]$ and $q$ are coprime. Moreover, the field extension $L K(x) / K(x)$ is radicial and, by [3, Chapitre V, $\S 8$, Proposition 6], its degree is a power of $p$. Consequently $[L: K]$ and $q$ are coprime hence $K^{\prime}$ satisfies condition (1). By definition, $K^{\prime}$ is the smallest perfect subfield of $\overline{K_{q}}$ containing $K_{q}$ so it satisfies condition (2).

By [14, Chapitre II, §4.1], $G_{K^{\prime}}$ can be identified to a subgroup of $G_{K_{q}}$ and its index is equal to the separability degree $\left[K^{\prime}: K_{q}\right]_{s}=1$. Thus, $G_{K^{\prime}}=G_{K_{q}}=S_{q}\left(G_{K}\right)$ is a pro- $q$-group and condition (3) follows. Finally, $\operatorname{cd}\left(K^{\prime}\right)=\operatorname{cd}_{q}(K)$ by [14, Chapitre I, $\S 3.3$, Corollaire 1].

\section{Proofs of the triviality results}

\subsection{Case where the index is prime to the characteristic}

We now come to the proof of Theorem 1.1.

Theorem Let $K$ be a field of characteristic $p$ (which can be zero) and $q$ be a prime number different from $p$. Suppose that $\operatorname{cd}_{q}(K) \leq 2$ and that $A$ is a central simple algebra over $K$ whose index is q-primary. Then the Whitehead groups $\operatorname{SK}_{1}(A)$ and $\operatorname{USK}_{1}(A)$ are trivial.

Proof. It suffices to prove the statement for division algebras of $q$-primary degree (see Section 2). Suppose that $D$ is such an algebra.

We first show that the reduced Whitehead group of $D$ is trivial. Let $b \in \mathrm{SL}_{1}(D)$. By Proposition 4.4, there exists a perfect field $L$ which is a filtered union of field extensions whose degree is prime to $q$ and such that $\operatorname{cd}(L) \leq 2$. By Corollary $3.2, L$ is thus a $C_{2}^{0}$-field. By Theorem 2.6, as $b \otimes 1 \in \mathrm{SL}_{1}\left(D \otimes_{K} L\right)$, one deduce that $b \otimes 1 \in\left[\left(D \otimes_{K} L\right)^{*},\left(D \otimes_{K} L\right)^{*}\right]$. We can suppose that $b \otimes 1 \in\left[\left(D \otimes_{K} K_{i}\right)^{*},\left(D \otimes_{K} K_{i}\right)^{*}\right]$ where $\left[K_{i}: K\right]$ and $q$ are coprime. On the one hand, by Lemma 4.1, it follows that $b^{\left[K_{i}: K\right]} \in\left[D^{*}, D^{*}\right]$. On the other hand, $b^{\operatorname{deg} D} \in\left[D^{*}, D^{*}\right]$ (see Subsection 2.1). Finally, the degree of $D$ being $q$-primary, $b \in\left[D^{*}, D^{*}\right]$, thus proving that $\operatorname{SK}_{1}(D)=1$.

Next we show that the reduced unitary Whitehead group of $D$ is trivial. Let $\sigma$ be an unitary involution over $D$. If $q$ is odd, $\operatorname{USK}_{1}(D) \hookrightarrow \operatorname{SK}_{1}(D)$ by $\operatorname{Lemma}_{2.10}$, so $\operatorname{USK}_{1}(D)=1 . \operatorname{Suppose}$ now that $q=2$ and that $p=\operatorname{char}(K) \neq 2$. Again, we apply Proposition 4.4 to find a perfect field $L$ which is a filtered union of field extensions of $F$ of odd degree with $\operatorname{cd}(L) \leq 2$. Let $L=\bigcup_{i \in I} F_{i}$ be such that $\left[F_{i}: F\right]$ is odd. For $i \in I$, let $E_{i}=K F_{i}$ and $E=\bigcup_{i \in I} E_{i}$. Thus $\left[E_{i}: F_{i}\right]=2$ and $\left[E_{i}: K\right]$ is odd for every $i \in I$ which means that $E$ is also a filtered union of field extensions of $K$ of odd degree. Moreover $E / L$ is a quadratic field extension with nontrivial automorphism $\tau$ and $D \otimes_{K} E$ is a central simple algebra over $E$ which is endowed with the unitary involution $\sigma \otimes \tau$. Let $a \in \Sigma^{\prime}(D)$. Then $a \otimes 1 \in \Sigma^{\prime}\left(D \otimes_{K} E\right)$. The field $L$ is a $C_{2}^{0}$-field by Corollary 3.2, so we can apply Theorem 2.11 to obtain that $a \otimes 1 \in \Sigma\left(D \otimes_{K} E\right)$. We can suppose that there exists $j \in I$ such that $a \otimes 1 \in \Sigma\left(D \otimes_{K} E_{j}\right)$ and, by Lemma 4.3 , we deduce that $a^{\left[E_{j}: K\right]} \in \Sigma(D)$. Last, let

$$
b=a^{\operatorname{deg} D}\left(\operatorname{Nrd}_{D / K}(a)\right)^{-1} .
$$

One checks that $b \in \mathrm{SL}_{1}(D)$ so $b^{\operatorname{deg} D} \in\left[D^{*}, D^{*}\right]$. By Proposition 2.9, $\left[D^{*}, D^{*}\right] \subset \Sigma(D)$ and $a^{\operatorname{deg} D^{2}} \in \Sigma(D)$. The degree of $D$ being even, $\operatorname{USK}_{1}(D)=1$. 
Remark 5.1. The proof of the previous Theorem for $\operatorname{USK}_{1}(A)$ in the case where $q \neq 2$ can be obtained independently from considerations about $\mathrm{SK}_{1}(A)$ by applying Lemma 4.3.

As an application, we obtain the following triviality result for $\mathrm{K}_{1}$ Spin:

Corollary 5.2. Let $K$ be a field of characteristic different from 2 satisfying $\operatorname{cd}_{2}(K) \leq 2$. If $A$ is a central simple algebra over $K$, then $\mathrm{K}_{1} \operatorname{Spin}(A)=1$.

Proof. This proof is analog to the case where the field is supposed to be a $C_{2}^{0}$-field. Suppose that $\sigma$ is a symplectic involution over $A$. In this case, it is well-known that the index of $A$ must be a power of 2 (see [7, Corollary 2.8]). Let $a \in R(A)$ and $\operatorname{Nrd}_{A / K}(a)=\alpha^{2}, \alpha \in K^{*}$. By Theorem 3.1 , there exists $b \in A^{*}$ such that $\operatorname{Nrd}_{A / K}(b)=\alpha$ and we can write $a=b^{2} c$ with $c \in \operatorname{SL}_{1}(A)$. By Theorem 1.1, $c \in\left[A^{*}, A^{*}\right]$. It suffices to show that $b^{2} \in \Sigma(A)\left[A^{*}, A^{*}\right]$. By [7, Proposition 4.17], there exists $g \in A^{*}$ such that the involution $\operatorname{Int}(g) \circ \sigma$ leaves $b$ invariant. Finally

$$
b \sigma(b)=b^{2}\left(b^{-1} g b g^{-1}\right) \in\left[A^{*}, A^{*}\right]
$$

thus $\mathrm{K}_{1} \operatorname{Spin}(A)=1$.

\subsection{Case where the index is a power of the characteristic}

We want to state analogs of Theorem 1.1 and Corollary 5.2 in the case where the index of $A$ is a power of the characteristic of $K$. To do this, we need the notion of separable $p$-dimension introduced by Gille and refer to [6] for precise results. This notion is a separable version of the $p$-dimension initially defined by Kato. More precisely:

Definition 5.3. Let $p$ be a prime number. The separable $p$-dimension of $K$ denoted by $\operatorname{dim}_{\mathrm{p}}^{\mathrm{sep}}(K)$ is defined as follows. If $p \neq \operatorname{char}(K)$, then $\operatorname{dim}_{\mathrm{p}}^{\mathrm{sep}}(K)=\operatorname{cd}_{p}(K)$. If $p=\operatorname{char}(K)$ then

$$
\operatorname{dim}_{\mathrm{p}}^{\mathrm{sep}}(K)=\inf \left\{r \geq 0 \mid H_{p}^{r+1}\left(K^{\prime}\right)=0 \text { for every finite separable field extension } K^{\prime} / K\right\},
$$

where $H_{p}^{r+1}\left(K^{\prime}\right)$ is the corresponding Kato cohomology group of $K^{\prime}$. The supremum of the separable $p$-dimensions of $K$ where $p$ runs over all prime numbers is called the separable dimension of $K$.

The following Theorem extends Merkurjev and Suslin's result 3.1 to every prime number:

Theorem 5.4 (Gille). Let $K$ be a field and $p$ be a prime number. Then, the following are equivalent:

(1) $\operatorname{dim}_{\mathrm{p}}^{\mathrm{sep}}(K) \leq 2$.

(2) For any finite separable field extension $E / K$ and for any central simple algebra $A$ over $E$, if the degree of $A$ is a power of $p$ then the reduced norm map $\operatorname{Nrd}_{A / K}: A^{*} \rightarrow E^{*}$ is surjective.

Proof. See [6, Theorem 7].

We can now be more precise about the missing cases in Theorem 1.1 and Corollary 5.2: 
Corollary 5.5. (1) Let $K$ be a field of characteristic $p \neq 0$. Suppose that $\operatorname{dim}_{\mathrm{p}}^{\mathrm{sep}}(K) \leq 2$ and that $A$ is a central simple algebra over $K$ whose index is p-primary. Then the Whitehead groups $\mathrm{SK}_{1}(A)$ and $\mathrm{USK}_{1}(A)$ are trivial.

(2) Let $K$ be a field of characteristic 2. Suppose that $\operatorname{dim}_{2}^{\mathrm{sep}}(K) \leq 2$ and that $A$ is a central simple algebra over $K$. Then $\mathrm{K}_{1} \operatorname{Spin}(A)=1$.

Proof. (1) The result is obtained by mimicking Yanchevski1's proofs of these facts over $C_{2}^{0}$-fields (see [20] and [19]) and using Theorem 5.4.

(2) The proof is analog to Corollary 5.2 using (1) and Theorem 5.4.

\subsection{Global results}

We can globalize all the previous results by stating:

Corollary (1) Suppose that $K$ is a field whose separable dimension is at most 2 . Then for any central simple algebra $A$ over $K$, the Whitehead groups $\operatorname{SK}_{1}(A)$ and $\operatorname{USK}_{1}(A)$ are trivial.

(2) Suppose that $K$ is a field whose separable 2-dimension is at most 2. Then for any central simple algebra $A$ over $K$, the Whitehead group $\mathrm{K}_{1} \operatorname{Spin}(A)$ is trivial.

Proof. It comes from Theorem 1.1 and Corollary 5.5.

Remarks 5.6. (1) Note that, in (1), the assertion is still true under the weaker hypothesis that $\operatorname{cd}(K) \leq 2$ if we suppose further that the index of $A$ is prime to the characteristic of $K$.

(2) In [24], Yanchevskiu has proved that if $\operatorname{char}(K)=0$ and if $\operatorname{vcd}(K):=\operatorname{cd}(K(\sqrt{-1})) \leq 2$ then $\operatorname{SK}_{1}(A)=\operatorname{USK}_{1}(A)=\mathrm{K}_{1} \operatorname{Spin}(A)=1$ for every central simple algebra $A$ over $K$.

\section{References}

[1] E. Bayer-Fluckiger, R. Parimala : Galois cohomology of the Classical groups over fields of cohomological dimension $\leqq 2$, Invent. Math. 122 (1995), 195-229.

[2] E. Bayer-Fluckiger, J.-P. Serre : Torsions quadratiques et bases normales autoduales, Amer. J. Math. 116 (1994), 1-63.

[3] N. Bourbaki : Corps commutatifs, dans Algèbre, Hermann, Paris, 1950, Chapitre 5.

[4] P. K. Draxl : Corps gauches à involution de deuxième espèce, SMF Astérisque 61 (1979), 63-72.

[5] P. K. Draxl : Skew fields, London Mathematical Society Lecture Note Series, vol. 81, Cambridge University Press, Cambridge, 1983.

[6] P. Gille : Invariants cohomologiques de Rost en caractristique positive (French) [Rost's cohomological invariants in positive characteristic] $K$-Theory 21 (2000), $\mathrm{n}^{\circ} 1,57-100$.

[7] M.-A. Knus, A. S. Merkurjev, M. Rost, J.-P. Tignol : The book of involutions, Coll. Pub. 44. Providence, RI : Amer. Math. Soc. (1998). 
[8] A. S. MerkurJev : K-theory of simple algebras, Proc. of Symposia in Pure Math., $\mathbf{5 8 . 1}$ (1995), 65-83.

[9] A. P. Monastyrny Akad. Nauk SSSR Ser. Mat. 54 (1990), no. 1, 60-96, 221; english translation : Math. USSRIzv. 36 (1991), no. 1, 61-100

[10] T. Nakayama, Y. Matsushima : Über die multiplikative Gruppe einer $p$-adischen Divisionsalgebra, Proc. Imp. Acad. Tokyo 19 (1943), 622-628.

[11] V. P. Platonov : On the Tannaka-Artin problem, Dokl. Akad. Nauk SSSR 221 (1975), 1038-1041 ; english translation : Soviet Math. Dokl. 16 (1975).

[12] V. P. Platonov, V. Yanchevski : : The Kneser-Tits conjecture for unitary groups, Dokl. Akad. Nauk USSR 225 (1975), 48-51; english translation : Soviet Math. Dokl. 16 (1975).

[13] V. P. Platonov, V. Yanchevskil : Finite-dimensional division algebras, Algebra IX, Finite groups of Lie Type, Finite-Dimensional Division Algebras (Berlin) (A. I. Kostrikin and I. R. Shafarevich, eds.), Encyclopaedia of Mathematical Science, vol. 77, Springer-Verlag, $121-233$.

[14] J.-P. Serre : Cohomologie galoisienne, Lecture notes in Mathematics 5, Springer-Verlag 1994.

[15] A. A. SusLin : Algebraic $K$-theory and the norm residue homomorphism, J. Soviet. Math. 30 (1985), 2556-2611.

[16] A. A. Suslin : $\mathrm{SK}_{1}$ of division algebras and Galois cohomology, Adv. in Soviet Math. 4 (1991), 75-99.

[17] J. Tits : Groupes de Whitehead de groupes algèbriques simples sur un corps, Séminaire Bourbaki, année 29, exposé nº 505, 1976/1977.

[18] S. Wang : On the commutator group of a simple algebra, Amer. J. Math. 72 (1950), $323-334$.

[19] V. Yanchevskil : Simple algebras with involution and unitary groups, Math. Sbornik 93 (1974) ; english translation : Math. USSR Sbornik, 22 (1974), 372-384.

[20] V. Yanchevskil : The commutator subgroups of simple algebras with surjective reduced norms, Dokl. Akad. Nauk SSSR 221 (1975) ; english translation : Soviet Math. Dokl. 16 (1975), 492-495.

[21] V. Yanchevskil : Reduced unitary $K$-theory and division rings over discretely valued Hensel fields, Izv. Akad. Nauk USSR Ser. Math. 42 (1978), 879-918 ; english translation : Math. USSR Izv. 13 (1979), 175-213.

[22] V. Yanchevskil : Reduced unitary $K$-theory, Applications to algebraic groups, Mat. Sbornik 110 (152) (1979), 579-596 ; english translation : Math. USSR Sbornik 38 (1981). 
[23] V. YAnchevskil : Symmetric and skew-symmetric elements of involutions, associated groups and the problem of decomposability of involutions, Proc. of Symposia in Pure Math. $\mathbf{5 8 . 2}$ (1995), 431-444.

[24] V. YAnchevskil : Whitehead groups and groups of $R$-equivalence classes of linear algebraic groups of non-commutative classical type over some virtual fields, Algebraic groups and arithmetic, 491-505, Tata Inst. Fund. Res., Mumbai, 2004.

Division of Pure Mathematics, School of Mathematical Sciences, University of Nottingham, University Park, Nottingham NG7 2RD, UK

E-mail address: grenier@math.univ-fcomte.fr 